\title{
Valores pessoais e organizacionais em diferentes grupamentos geracionais
}

\section{Personal and organizational values in different generational clusters}

\author{
Alice Maria Garcia de Mello ${ }^{1}$ \\ Anderson de Souza Sant'Anna²
}

\section{Resumo}

Este artigo apresenta resultados de uma pesquisa destinada à investigação de relações entre os construtos Valores Pessoais (VP) e Valores Organizacionais (VO), em distintos grupamentos geracionais. Para a operacionalização do estudo, foram utilizados o Inventário de Valores e o Inventário de Perfis de Valores Organizacionais. Quanto à coleta de dados, o estudo compreendeu 276 participantes de programas de desenvolvimento de executivos. Como resultados, identificaram-se diferenças estatisticamente significativas em relação aos valores pessoais associados a poder, realização e universalismo. Com relação aos VO, os resultados apontaram diferenças para "bem-estar", "domínio" e "prestígio". Já a análise das relações entre VP e VO indicou diferenças entre os pares geracionais investigados, concentradas nos valores pessoais "autodeterminação", "benevolência", "hedonismo" e "tradição", quando comparados aos $\mathrm{VO}$ pesquisados.

Palavras-chave: Valores. Valores Pessoais. Valores Organizacionais. Diversidade. Diversidade Geracional.

\section{Abstract}

This paper aims to investigate relations between the constructs Personal Values (PV) andOrganizational Values (OV) in different generational groups. Foroperationalization of the study were used Values Inventory Scale, and Values Organizational Profiles

\footnotetext{
1 Mestre em Administração pela Pontifícia Universidade Católica de Minas Gerais. Professora e Gerente de Programas de Desenvolvimento de Executivos da Fundação Dom Cabral - Brasil E-mail: alice@fdc.org.br

2 Pós-doutor em Teoria Psicanalítica pela Universidade Federal do Rio de Janeiro. Doutor em Administração pela Universidade Federal de Minas Gerais. Professor do Programa de Mestrado Profissional em Administração e Coordenador do Núcleo de Desenvolvimento de Pessoas e Liderança da Fundação Dom Cabral - Brasil - E-mail: anderson@fdc.org.br
} 
Scale. As to data collection, the study comprised 276 Brazilians Executive Development Programs participants. The results identified significant statistical differences regarding the personal values: Power, Achievement, and Universalism. Concerning OV, the results pointed out differences on Well-being, Domain and Prestigious. Besides, analysis of relations between PV and OV indicated differences between the generational peers investigated concentrated on the personal values Self-determination, Goodwill, Hedonism, and Tradition, when compared to those reported to the OV investigated.

Keywords: Values.Personal Values.Organizational Values.Diversity. GenerationalDiversity.

\section{Introdução}

A importância das diferenças entre as gerações é destacada por Zemke, Raines e Filipczak (2000), ao afirmarem que elas são originadas de diferentes valores, atitudes, ambições e modelos mentais podem aportar novas competências e perspectivas de análise, constituindose em relevantes fatores de inovação, diferenciação e vantagens competitivas. Daí o interesse crescente por melhor mensurar as expectativas e valores dos distintos grupos geracionais, que pela primeira vez podem ser identificados atuando de forma conjunta nos mesmos ambientes laborais. De fato, não é mais tão incomum quatro gerações interagindo, simultaneamente: geração veteranos, geração pós-guerra (baby boomers), geração $\mathrm{X}$ e geração $\mathrm{Y}$.

De acordo com Gouveia et al. (2001), Pasquali e Alves (2004) e Rohan (2000), Rokeach (1973) foi o primeiro autor a apresentar um instrumento destinado à mensuração de valores como um construto específico, demonstrando sua centralidade no sistema cognitivo humano. No entanto, Schwartz (1992) aportou ao contexto organizacional possibilidades de investigação sistemática dos valores humanos, conforme atualmente adotada.

É interessante observar que, no Brasil, diferentemente de outros países, não é hábito se utilizar um mesmo instrumento para mensuração de valores organizacionais e pessoais. Eles, na verdade, são entendidos 
como conceitualmente distintos, resultando em instrumentos igualmente específicos para sua investigação (Oliveira; Tamayo, 2004). Nesse sentido, para a análise dos valores organizacionais, identifica-se, com maior frequência, a aplicação do Inventário de Perfis de Valores Organizacionais (IPVO), na versão validada por Oliveira e Tamayo (2004). Já para a tangibilização dos valores pessoais, é comumente adotado o Schwartz Value Survey (SVS), desenvolvido por Schwartz (1992) e adaptado para o Brasil por Tamayo e Schwartz (1993).

Cabe salientar, ademais, o número crescente de pesquisas nacionais buscando relacionar valores a outros elementos do comportamento organizacional, tais como comprometimento (Tamayo, 2005), cidadania organizacional (Porto, Tamayo, 2002; Maia, Oliveira, Ximenes, 2007; Tamayo et al., 1998), satisfação no trabalho (Tamayo, 2000), estresse ocupacional (Tamayo, 2001; 2002), conhecimento organizacional (Miguel; Teixeira, 2009) e qualidade de vida no trabalho (Dias; Del Maestro Filho; Moraes, 2006).

Concomitantemente, pesquisas mais sistemáticas sobre gerações têm-se feito notar no país, contribuindo para a ampliação da literatura científica sobre o tema (Coimbra, Schikmann, 2001; Dunzer, Melo, Melo, 2008; Ferreira, 2008; Pedrosa, Sant'anna, 2003; Santos, Abrahim, 2008; Sarsur, Toledo, 2006; Veloso, Dutra, Nakata, 2008). Na literatura internacional, igualmente, os estudos sobre o tema avançam, com destaque para relações do construto com desempenho organizacional (Halliman, 1998); liderança (Yu; Miller, 2004); comprometimento, aprendizagem e retenção de talentos (D’Amato; Herzfeldt, 2008); carreira (Dries; Pepermans; Kerpel, 2008) e motivação (Montana, Petit, 2008; Wong et al., 2008).

Nesse contexto, este estudo visa contribuir com a cadeia monológica de estudos sobre o tema, investigando relações entre os construtos Valores Pessoais (VP) e Valores Organizacionais (VO), em distintos grupamentos geracionais que coexistem em ambientes organizacionais contemporâneos. Assim sendo, a questão central que orientou sua condução pode ser assim sintetizada: em que medida 
podem se observar diferenças significativas entre os construtos VP e VO nas gerações que hoje coexistem nos ambientes organizacionais?

Com vistas a respondê-la, buscou-se: 1) identificar os valores pessoais e organizacionais, segundo a percepção de diferentes grupamentos geracionais que hoje coabitam distintos espaços ocupacionais, com destaque para a análise de suas semelhanças e diferenças; 2) investigar semelhanças e diferenças entre valores pessoais e organizacionais, considerando pares geracionais comumente identificados na literatura: geração $\mathrm{Y}$ e geração baby boomers (Y-BB); geração $X$ e geração baby boomers (X-BB); geração $X$ e geração $Y(X-Y)$.

Em termos metodológicos, a pesquisa que subsidiou a realização deste estudo pode ser caracterizada como de natureza quantitativa, conduzida por meio da técnica de survey.

Finalmente, quanto à sua relevância, a opção por este estudo vincula-se, em termos práticos, ao momento vivenciado pelas organizações no que tange ao desafio de lidar com a diversidade derivada das mudanças demográficas em curso, requerendo novas estratégias de mediação e antecipação de conflitos. Nessa direção, pretende contribuir também, em termos teóricos para a ampliação da compreensão dos construtos em análise, por meio de análise mais sistemática.

Vislumbra-se que os dados deste estudo poderão apresentar novos subsídios às áreas de recursos humanos na formulação de políticas e práticas de gestão mais afins às transformações socioeconômicodemográficas em curso.

\section{Fundamentação teórica}

Segundo Arsenault (2004), a composição da força de trabalho nunca foi tão diversificada quanto hoje, representada por um mix de etnias, gêneros, classes sociais, formações, geografias e culturas. Tal fenômeno tem despertado as empresas para o potencial e para os riscos associados à intensa diversidade. Para o autor, não obstante, 
pouca atenção tem sido direcionada a estudos mais sistemáticos sobre a diversidade geracional, abrindo espaços para estereótipos, tanto na mídia quanto na academia e nas organizações.

A temática das diferenças entre gerações é também ressaltada por Zemke, Raines e Filipczak (2000), ao afirmarem que elas guardam suas origens em diferentes valores, atitudes, ambições e modelos mentais, os quais as pessoas trazem consigo. Em outros termos, a constituição de uma geração está relacionada a fatores que incluem atitudes, valores e crenças sobre fatores sócio-histórico-culturais, tais como a família, religião e estilo de vida (Strauss; Howe, 1997).

A noção de geração é também abordada por Beck (2001), para quem uma geração é constituída por indivíduos nascidos em um mesmo período de tempo, recebendo as mesmas influências históricas e culturais relativas àquele determinado período histórico. Já Cennamo e Gardner (2008) a definem como um grupo que pode ser identificado não só pelo ano de nascimento como também por eventos significativos em fases críticas do desenvolvimento da vida de um grupo. García-Lombardia, Stein e Pin (2008), por sua vez, conceituam geração como um grupo de representantes de determinada faixa etária que compartilha, ao longo de sua vida, um conjunto de experiências que o diferencia de seus antecessores. Nessa mesma linha de raciocínio, Sullivan et al. (2009) afirmam que os indivíduos de uma geração podem ser diferenciados de outros não só pela faixa etária, mas também por suas experiências sociais e históricas, que influenciarão para sempre suas características.

Visando estabelecer determinantes constituintes de uma geração, Wyatt (1993) aponta para cinco fatores desencadeadores: 1) eventos traumáticos, como o assassinato de um líder político ou conflitos armados de grandes proporções; 2) mudanças demográficas que influenciam a distribuição de recursos em uma sociedade; 3) fatos relevantes que conectam uma geração a um ciclo de prosperidade ou crise; 4) criação de espaços sagrados, como Woodstock, sustentando a memória coletiva; 5) existência de líderes que se destacam por seu trabalho. 
A relevância de influências históricas e culturais na diferenciação entre as gerações também é compartilhada por Hess e Jepsen (2009), segundo os quais cada geração pode ser percebida a partir de um padrão único de comportamento, baseado no compartilhamento de suas experiências.

Considerando contribuições aportadas por Hess e Jepsen (2009), e Wyatt (1993), diversos autores buscaram a construção de tipologias geracionais. Smith e Clurman (1997) vão então desenvolver um modelo operativo compreendendo três grupamentos: os maduros ou veteranos, os baby boomers e a geração X. Zemke, Raines e Filipczak (2000) acrescentam um quarto grupo, a geração $\mathrm{Y}$.

Não obstante a ausência de consenso quanto às denominações atribuídas às gerações e sua universalidade, a revisão de literatura indica como mais reiteradas denominações envolvendo quatro gerações majoritariamente presentes no panorama globalizado atual: veteranos, baby boomers, geração $\mathrm{X}$ e geração $\mathrm{Y}$, cujas características são apresentadas, de forma sintetizada, na Figura 1. 


\section{Figura 1 - Representações geracionais}

\begin{tabular}{|c|c|c|c|c|}
\hline GERAÇÕES & $\begin{array}{l}\text { VETERANOS } \\
1922-1945\end{array}$ & $\begin{array}{c}\text { BABY } \\
\text { BOOMERS } \\
1946-1961\end{array}$ & $\begin{array}{l}\text { GERAÇÃO X } \\
\text { 1962-1979 }\end{array}$ & $\begin{array}{c}\text { GERAÇÃO Y } \\
\text { A partir dos anos } 1980\end{array}$ \\
\hline $\begin{array}{l}\text { Outras } \\
\text { denominações }\end{array}$ & $\begin{array}{l}\text { Maduros, } \\
\text { Geração do } \\
\text { Silêncio (Kirk, } \\
\text { 2002; Smith, } \\
\text { Clurman, 1997; } \\
\text { Sullivan et al., } \\
\text { 2009). }\end{array}$ & $\begin{array}{l}\text { Pós-Guerra } \\
\text { (Smith; } \\
\text { Clurman, 1997). }\end{array}$ & $\begin{array}{l}\text { Geração de } \\
\text { Preguiçosos } \\
\text { (Hankin, 2004; } \\
\text { Mcdonald, Hite, 2008; } \\
\text { Smith, Clurman, } \\
\text { 1997). }\end{array}$ & $\begin{array}{l}\text { Why Generation, Millennial } \\
\text { Generation, MySpace } \\
\text { Generation, The Nexters, } \\
\text { Internet Generation e Greater } \\
\text { Generation (Kick, 2002; } \\
\text { Clurman, 1997; Sullivan et al., } \\
\text { 2009). }\end{array}$ \\
\hline Características & $\begin{array}{l}\text { Disciplina, } \\
\text { valorização do } \\
\text { trabalho, ética, } \\
\text { lealdade às } \\
\text { organizações. } \\
\text { (Hankin, 2004; } \\
\text { Sullivan et al., } \\
\text { 2009). }\end{array}$ & $\begin{array}{l}\text { Viver para } \\
\text { trabalhar, busca } \\
\text { do desafio } \\
\text { profissional, } \\
\text { valorização } \\
\text { do sucesso } \\
\text { e conforto } \\
\text { material, } \\
\text { respeito à } \\
\text { autoridade e } \\
\text { à hierarquia, } \\
\text { gosto pelo } \\
\text { comando } \\
\text { (Gursoy et } \\
\text { al., 2008; } \\
\text { Kupperschmidt, } \\
2000 ; \text { Sullivan } \\
\text { et al., 2009). }\end{array}$ & $\begin{array}{l}\text { Trabalhar para viver, } \\
\text { maior importância } \\
\text { à sua carreira do } \\
\text { que à organização, } \\
\text { valorização do } \\
\text { desenvolvimento de } \\
\text { habilidades, equilíbrio } \\
\text { entre vida pessoal e } \\
\text { profissional, menor } \\
\text { valorização do status, } \\
\text { menor lealdade } \\
\text { às organizações, } \\
\text { lealdade aos grupos } \\
\text { de trabalho e às } \\
\text { chefias, aversão à } \\
\text { hierarquia, maior } \\
\text { flexibilidade para } \\
\text { as mudanças, } \\
\text { mobilidade, foco } \\
\text { na produtividade; } \\
\text { meritocracia, rápido } \\
\text { reconhecimento } \\
\text { e promoção, } \\
\text { autoconfiança } \\
\text { (Cennamo\&Gardner, } \\
\text { 2008; Gursoy et al., } \\
\text { 2008; Sullivan et al., } \\
2009 \text { ). }\end{array}$ & $\begin{array}{l}\text { Intimidade com a tecnologia, } \\
\text { uso das redes sociais, vivência } \\
\text { em diferentes contextos } \\
\text { internacionais, valorização do } \\
\text { equilíbrio entre vida pessoal } \\
\text { e profissional, estilo de vida e } \\
\text { desenvolvimento na carreira. } \\
\text { Independentes, confiantes, } \\
\text { determinados, otimistas, } \\
\text { idealistas, alta expectativa } \\
\text { em relação a si mesmos, } \\
\text { orientação para objetivos } \\
\text { e metas. Pensamento } \\
\text { empreendedor, senso de } \\
\text { responsabilidade, feedback } \\
\text { imediato, senso de urgência } \\
\text { para as realizações, gostam } \\
\text { dos desafios do trabalho e } \\
\text { novas oportunidades. Criativos, } \\
\text { valorizam a liberdade e a } \\
\text { flexibilidade. } \\
\text { Menor lealdade às } \\
\text { organizações, menos valor à } \\
\text { segurança no emprego como } \\
\text { um fator determinante do } \\
\text { mercado de trabalho. } \\
\text { Flexíveis às mudanças, } \\
\text { valorizam o desenvolvimento de } \\
\text { habilidades, sociáveis, decidem } \\
\text { e agem com responsabilidade } \\
\text { (Cennamo, Gardner, 2008; } \\
\text { Chen, Choi, 2008; Martin, 2005; } \\
\text { Montana, Petit, 2008; Sullivan } \\
\text { et al., 2009; Wong et al., 2008). }\end{array}$ \\
\hline $\begin{array}{l}\text { Eventos } \\
\text { históricos }\end{array}$ & $\begin{array}{l}\text { Grande } \\
\text { Depressão, } \\
\text { Pearl Harbor e a } \\
\text { Segunda Guerra } \\
\text { Mundial } \\
\text { (Hankin, 2004; } \\
\text { Sullivan et al., } \\
\text { 2009). }\end{array}$ & $\begin{array}{l}\text { Assassinatos } \\
\text { de JFK, Bobby } \\
\text { Kennedy e } \\
\text { Martin Luther } \\
\text { King, desilusão } \\
\text { com a Guerra } \\
\text { do Vietnã, } \\
\text { escândalo de } \\
\text { Watergate e } \\
\text { a renúncia do } \\
\text { Presidente } \\
\text { Nixon } \\
\text { (Sullivan et al., } \\
\text { 2009) }\end{array}$ & $\begin{array}{l}\text { Queda do Muro } \\
\text { de Berlim e o } \\
\text { desastre com a nave } \\
\text { Challenger } \\
\text { (Sullivan et al., 2009) }\end{array}$ & $\begin{array}{l}\text { Atentados de } 11 \text { de setembro } \\
\text { nos EUA, aumento da violência } \\
\text { nas escolas, escândalo } \\
\text { envolvendo Bill Clinton (então } \\
\text { presidente americano), Guerra } \\
\text { do Iraque (Dwyer, 2009; } \\
\text { Sullivan et al., 2009) }\end{array}$ \\
\hline
\end{tabular}

Fonte: Citadas na figura. 
Ressalta-se que, não raro, diferenças entre as gerações são confundidas com mudanças etárias, com o grau de experiência ou momentos nos ciclos de vida ou carreira (Cennamo; Gardner, 2008). Cennamo e Gardner (2008) ressaltam, no entanto, que relações entre tais grupamentos e diferenças em relação aos valores e significados conferidos ao trabalho, à composição das famílias, às expectativas em relação ao equilíbrio entre vida pessoal e profissional, ao aumento do uso da tecnologia e à busca pelo desenvolvimento contínuo de novas competências revelam-se mais fundamentais à análise desse fenômeno do que propriamente a idade cronológica.

\subsection{Valores}

O construto "valor" tem sido amplamente pesquisado em diversas disciplinas das Ciências Sociais, como a Antropologia, a Filosofia e a Sociologia (Rokeach, 1973), porém, sem maiores acordos quanto à sua conceituação e formas de mensuração (Kluckhohn, 1951; Rohan, 2000). No mundo organizacional, tal ausência de consenso (Meglino; Ravlin, 1998), aliada à centralidade do trabalho no mundo contemporâneo, amplifica sua relevância nos estudos organizacionais (Oliveira; Tamayo, 2004). Nessa direção, explicitam-se a seguir os marcos teóricos e os instrumentos de mensuração utilizados para a operacionalização deste estudo.

\subsubsection{Valores Pessoais}

Como marco teórico para a mensuração dos valores pessoais, fezse uso, para fins desta pesquisa, do Inventário de Valores de Schwartz (Schwartz Value Survey - SVS), desenvolvido por Schwartz (1992). Instrumento amplamente adotado e validado em mais de 60 países, de todos os continentes, tendo sido traduzido para 46 línguas.

Em linhas gerais, o SVS é composto por 57 itens, compreendendo cada um dos tipos motivacionais constantes da teoria de valores proposta pelo autor. Tais itens encontram-se agrupados em duas 
escalas. Uma primeira, composta por 30 itens, relaciona os objetivos e valores potencialmente desejáveis, em forma de substantivos; a outra contém 27 itens direcionados à mensuração, na forma de adjetivos, de formas potencialmente desejáveis de ação.

No Brasil, o instrumento foi validado por Tamayo e Schwartz (1993), contemplando a mensuração das seguintes categorias de valores: realização, benevolência, universalismo e poder. Tal versão, adotada para fins da pesquisa, subsidiou a realização deste estudo.

Definido o marco teórico para a mensuração dos valores pessoais, será apresentado a seguir o marco teórico utilizado para a avaliação dos valores organizacionais.

\subsubsection{Valores Organizacionais}

Para mensuração dos valores organizacionais, optou-se pelo Inventário de Perfis de Valores Organizacionais (IPVO), conforme desenvolvido por Oliveira e Tamayo (2004). A opção por tal instrumento deriva de sua maior simplicidade de aplicação, comparativamente ao Inventário de Valores Organizacionais (IVO), originalmente desenvolvido por Tamayo, Mendes, Paz (2000), permitindo ser aplicado a indivíduos com níveis mais baixos de escolaridade (Porto, 2005).

A versão do IPVO proposta por Oliveira e Tamayo (2004) é composta por 48 descrições de perfis de organizações, os quais devem ser avaliados por escala não numérica, variando de "é muito parecida com a minha organização" a "não se parece com minha organização", visando à mensuração de oito fatores: autonomia, bem-estar, realização, domínio, prestígio, tradição, conformidade e preocupação com a coletividade.

$\mathrm{Na}$ Figura 2 apresentam-se os fatores do IPVO e suas correspondências com os tipos motivacionais de valores, conforme utilizados para fins deste estudo. 
Figura 2 - Fatores do IPVO, correspondência com os tipos motivacionais de valores, metas dos valores organizacionais e coeficientes alpha

\begin{tabular}{|l|l|l|}
\hline FATORES & CORRESPONDÊNCIA & \multicolumn{1}{|c|}{ METAS } \\
\hline Autonomia & $\begin{array}{l}\text { Autodeterminação } \\
\text { Estimulação }\end{array}$ & $\begin{array}{l}\text { Oferecer desafios e variedade } \\
\text { no trabalho, estimular a } \\
\text { curiosidade, a criatividade e a } \\
\text { inovação. }\end{array}$ \\
\hline Bem-estar & Hedonismo & $\begin{array}{l}\text { Promover a satisfação, o bem- } \\
\text { estar e a qualidade de vida no } \\
\text { trabalho. }\end{array}$ \\
\hline Realização & Realização & $\begin{array}{l}\text { Valorizar a competência e o } \\
\text { sucesso dos trabalhos. }\end{array}$ \\
\hline Domínio & Poder & $\begin{array}{l}\text { Obter lucros, ser competitiva e } \\
\text { dominar o mercado. }\end{array}$ \\
\hline Prestígio & Poder & $\begin{array}{l}\text { Ter prestígio, ser conhecida } \\
\text { e admirada por todos, } \\
\text { oferecer produtos e serviços } \\
\text { satisfatórios para os clientes. }\end{array}$ \\
\hline Tradição & Tradição & $\begin{array}{l}\text { Manter a tradição e respeitar os } \\
\text { costumes da organização. }\end{array}$ \\
\hline Conformidade & Conformidade & $\begin{array}{l}\text { Promover a correção, a } \\
\text { cortesia e as boas maneiras no } \\
\text { trabalho e o respeito às normas } \\
\text { da organização. }\end{array}$ \\
\hline $\begin{array}{l}\text { com a } \\
\text { coletividade }\end{array}$ & $\begin{array}{l}\text { Promover a justiça e a } \\
\text { igualdade na organização, } \\
\text { bem como a tolerância, a } \\
\text { sinceridade e a honestidade. }\end{array}$ \\
\hline
\end{tabular}

Fonte: Oliveira e Tamayo (2004, p. 137).

Apresentam-se na seção seguinte os aspectos metodológicos orientadores da pesquisa que subsidiou os resultados deste estudo. 


\section{Aspectos metodológicos}

Este estudo pode ser caracterizado como uma pesquisa de natureza quantitativa, realizada por meio da técnica de survey. A pesquisa pode também ser caracterizada como de natureza descritiva. Quanto à abordagem, adotou-se o estudo de campo, que, segundo Mattar (2001), é indicado e deve ser utilizado quando o interesse for o conhecimento do inter-relacionamento entre as diversas variáveis que ocasionam um fenômeno, principalmente quando for difícil entender o fenômeno sem a compreensão desse inter-relacionamento.

Com relação ao seu público-alvo, o estudo foi realizado com participantes de programas de desenvolvimento de executivos da Fundação Dom Cabral. A escolha dessa instituição se deu pela heterogeneidade de seu público, tanto em relação à presença de empresas de diferentes setores da economia e segmentos de atuação quanto à diversidade de seu portfolio de programas e participantes de diferentes grupamentos geracionais.

Foi adotado como instrumento de coleta de dados um questionário composto por escalas do tipo Likert de nove pontos para o instrumento Schwartz Value Survey (SVS), e de seis pontos para o Inventário de Perfis de Valores Organizacionais (IPVO), estruturadas em três blocos. Um primeiro direcionado à investigação de dados pessoais e profissionais dos respondentes. Já os blocos 2 e 3 tiveram como foco avaliar os valores pessoais e organizacionais por meio do SVS e IPVO, abrangendo um total de 113 itens.

Como estratégia de coleta de dados, após autorização da instituição e disponibilização aos pesquisadores da relação de seus programas e participantes, foram encaminhados, via e-mail, 405 questionários, tendose obtido, ao final, um total de 276 instrumentos válidos.

No que tange ao tratamento dos dados coletados, foram aplicadas estatísticas descritivas e multivariadas. Para tal, procedeuse, inicialmente, à verificação de pressupostos à análise multivariada: normalidade, linearidade, multicolinearidade, bem como análise dos 
outliers, uni e multivariados. Isto posto, foi procedida a análise da dimensionalidade dos construtos investigados (Netemeyer; Bearden; Sharma, 2003).

Na sequência, procedeu-se à análise fatorial exploratória e à análise da confiabilidade das escalas, por meio do cálculo da validade convergente. Para esse fim, recorreu-se ao procedimento proposto por Bagozzi, Yi e Philips (1991), os quais sugerem que a Análise Fatorial Confirmatória (AFC) seja a ferramenta empregada para analisar se os indicadores se correlacionam significativamente aos construtos. O método proposto consistiu em comparar a significância das cargas fatoriais dos construtos ao valor crítico da estatística $t$ unicaudal, considerando um nível de $5 \%$ ou $1 \%$ de significância, tendo os valores $t$ das cargas fatoriais apresentado valores superiores a 1,65

Neste estudo, a significância das cargas foi obtida por meio da estimação por mínimos quadrados parciais (Chin, 1998). Deve-se ressaltar que esses métodos de estimação da significância partem da análise de reamostragem (bootstrapping), portanto, são estimativas empíricas, e não estimativas teóricas de erro padrão.

Outra medida de confiabilidade empregada foi a variância média extraída (Average Variance Extracted - AVE), desenvolvida por Fornell e Larcker (1981). Essa medida representa a confiabilidade média dos indicadores e tem como vantagem não ser superestimada no caso de construtos com maior número de indicadores, além de refletir melhor a confiabilidade que seria possível obter de um indicador isolado da pesquisa. Como pontos de corte para essas medidas, empregou-se o valor de 0,700 para a Confiabilidade Composta (CC) e 0,400 para AVE, conforme Tabela 1. 
Tabela 1. Medidas de confiabilidade dos indicadores

\begin{tabular}{lccc}
\hline ESCALAS & AVE & $\begin{array}{c}\text { CONFIABILIDADE } \\
\text { COMPOSTA }\end{array}$ & $\begin{array}{c}\text { ALPHA DE } \\
\text { CRONBACH }\end{array}$ \\
\hline Autodeterminação (Pessoal) & 0,37 & 0,78 & 0,69 \\
Benevolência (Pessoal) & 0,46 & 0,78 & 0,62 \\
Conformidade (Pessoal) & 0,41 & 0,72 & 0,54 \\
Estimulação (Pessoal) & 0,75 & 0,86 & 0,68 \\
Hedonismo (Pessoal) & 0,61 & 0,82 & 0,68 \\
Poder (Pessoal) & 0,50 & 0,80 & 0,68 \\
Realização (Pessoal) & 0,40 & 0,77 & 0,66 \\
Segurança (Pessoal) & 0,36 & 0,73 & 0,57 \\
Tradição (Pessoal) & 0,40 & 0,73 & 0,50 \\
Universalismo (Pessoal) & 0,37 & 0,80 & 0,72 \\
Autonomia (Organizacional) & 0,53 & 0,90 & 0,87 \\
Bem-Estar (Organizacional) & 0,55 & 0,88 & 0,84 \\
Conformidade (Organizacional) & 0,49 & 0,79 & 0,67 \\
Domínio (Organizacional) & 0,48 & 0,85 & 0,78 \\
Preocupação com a Coletividade & 0,51 & 0,88 & 0,85 \\
(Organizacional) & 0,63 & 0,87 & 0,81 \\
Prestígio (Organizacional) & 0,54 & 0,85 & 0,78 \\
Realização (Organizacional) & 0,59 & 0,80 & \\
Tradição (Organizacional) & & & 0,73 \\
\hline
\end{tabular}

Fonte: Dados da pesquisa

Por fim, a análise da validade discriminante entre os construtos (Netemeyer; Bearden; Sharma, 2003) foi calculada indicando como resultado a validade discriminante segundo os critérios adotados.

Além disso, foi utilizado o software SmartPLS, destinado a testar o efeito das dimensões de valores pessoais sobre os valores organizacionais. Desse modo, criou-se um modelo de pesquisa em que aqueles foram considerados antecedentes destes.

Finalmente, cabe mencionar a não consideração, para efeito deste estudo, de representantes da geração veteranos, tendo em vista 
o reduzido número de respondentes desta geração, consistindo em uma das limitações deste estudo.

\section{Apresentação e discussão dos dados}

No que diz respeito às variáveis demográficas investigadas, tem-se que $68 \%$ dos respondentes são do sexo masculino e $32 \%$, do feminino. Quanto à faixa etária, tem-se a predominância de respondentes com idade entre 31 e 48 anos (72\%), sendo a média de idade de 39 anos, com desvio de 8,83 . Vale observar que apenas três respondentes tinham mais de 65 anos.

Em termos de adequação às tipologias recorrentes na literatura científica revisada, os dados apresentam clara predominância de membros da geração $X(72 \%)$, com igual percentual de participação de representantes da geração $Y(14 \%)$ e baby boomers (14\%). Haja vista a ausência de um número significativo de respondentes pertencentes à geração veteranos, não foi possível sua consideração para efeito das análises estatísticas propostas.

Quanto ao perfil profissional, à exceção de um respondente, todos se encontravam vinculados a organizações: $27 \%$ com 1 a 5 anos de empresa, 23\% com mais de 20 anos, $20 \%$ de 6 a 10 anos, $16 \%$ de 11 a 15 anos, e $8 \%$ há menos de 1 ano.

\subsection{Valores Pessoais}

Com relação aos tipos motivacionais de valores pessoais, quando analisada a geração baby boomers, composta por indivíduos na faixa etária entre 50 e 65 anos, os dados obtidos revelaram as seguintes prioridades axiológicas, em ordem decrescente de intensidade: benevolência $(6,07)$, autodeterminação $(5,74)$, universalismo $(5,61)$, conformidade $(5,58)$, hedonismo $(5,36)$, segurança $(5,34)$, realização $(4,88)$, estimulação $(4,37)$, tradição $(4,27)$ e poder $(3,90)$. 
Compreendendo os indivíduos na faixa etária entre 32 e 49 anos, identificados como geração $X$, as prioridades axiológicas corresponderam, em ordem decrescente a: benevolência $(6,08)$, autodeterminação $(5,80)$, hedonismo $(5,45)$, conformidade $(5,41)$, realização $(5,32)$, segurança $(5,25)$, universalismo $(5,25)$, estimulação $(4,65)$, tradição $(4,17)$ e poder $(4,09)$.

Já em relação à geração $\mathrm{Y}$, representada pelos indivíduos com menos que 31 anos, os resultados indicam, em ordem decrescente de intensidade: benevolência $(6,07)$, autodeterminação $(5,88)$, hedonismo $(5,75)$, realização $(5,55)$, conformidade $(5,43)$, segurança $(5,24)$, universalismo $(4,93)$, estimulação $(4,65)$, tradição $(4,42)$ e poder $(4,38)$.

Os dados da Tabela 2 apresentam os dados relativos aos tipos motivacionais de valores pessoais para o conjunto das gerações investigadas.

Tabela 2 - Médias dos tipos motivacionais de valores pessoais por grupos, com comparações de médias padronizadas

\begin{tabular}{lccc}
\hline & \multicolumn{3}{c}{ GRUPOS } \\
TIPOS MOTIVACIONAIS DE VALORES & A & B & Goomers \\
\cline { 2 - 4 } & Geração X & Geração Y \\
\hline Autodeterminação (P) & 5,74 & 5,80 & 5,88 \\
Benevolência (P) & 6,07 & 6,08 & 6,07 \\
Conformidade (P) & 5,58 & 5,41 & 5,43 \\
Estimulação (P) & 4,37 & 4,65 & 4,65 \\
Hedonismo (P) & 5,36 & 5,45 & 5,75 \\
Poder (P) & 3,90 & 4,05 & $4,38 a$ \\
Realização (P) & 4,88 & $5,32 a$ & $5,55 a$ \\
Segurança (P) & 5,34 & 5,25 & 5,24 \\
Tradição(P) & 4,27 & 4,17 & 4,42 \\
Universalismo (P) & $5,61 b c$ & $5,25 c$ & 4,93 \\
\hline
\end{tabular}

Fonte: Dados da Pesquisa 
Pelos dados da Tabela 2, constata-se a existência de diferenças estatisticamente significativas entre as três gerações investigadas no que se refere aos tipos motivacionais de valores pessoais de poder, realização e universalismo.

\subsection{Valores Organizacionais}

Neste subitem, são apresentados os resultados do IPVO para cada uma das gerações investigadas. Cabe salientar que, para a mensuração dos itens constantes desse instrumento, adotou-se uma escala Likert de seis pontos, variando de 1 a 6 , porém invertida, na qual quanto mais próximo de 1, maior ênfase atribuída ao item, e quanto mais próximo de 6 , menor a ênfase.

Como resultado, tem-se para a geração baby boomers as seguintes percepções quanto às prioridades axiológicas da organização, em ordem crescente: prestígio $(1,69)$, conformidade $(1,88)$, preocupação com a coletividade $(2,19)$, realização $(2,32)$, domínio $(2,49)$, autonomia $(2,69)$, bem-estar $(2,81)$ e tradição $(3,36)$.

Já segundo a percepção da geração X, as prioridades axiológicas da organização se apresentam, em ordem crescente de intensidade, da seguinte forma: prestígio $(1,90)$, domínio $(2,05)$, conformidade $(2,09)$, preocupação com a coletividade $(2,14)$, realização $(2,21)$, autonomia $(2,63)$, bem-estar $(3,20)$ e tradição $(3,31)$.

Quanto à geração $\mathrm{Y}$, as prioridades axiológicas da organização assim se revelam, considerando a ordem crescente de intensidade: domínio e prestígio $(2,09)$; conformidade e realização $(2,11)$; preocupação com a coletividade $(2,21)$, autonomia $(2,48)$, bem-estar $(3,04)$ e tradição $(3,42)$.

Na Tabela 3, são apresentados os dados relativos aos tipos motivacionais de valores organizacionais para o conjunto das gerações investigadas. 
Tabela 3 - Médias dos fatores por grupos, com comparações de médias padronizadas

\begin{tabular}{|c|c|c|c|}
\hline \multirow[b]{2}{*}{ CATEGORIAS } & \multicolumn{3}{|c|}{ GRUPOS } \\
\hline & $\begin{array}{c}\text { Baby Boomers } \\
\text { A }\end{array}$ & $\begin{array}{c}\text { Geração X } \\
\text { B }\end{array}$ & $\begin{array}{c}\text { Geração Y } \\
\text { C }\end{array}$ \\
\hline Autonomia $(\mathrm{O})$ & 2,69 & 2,63 & 2,48 \\
\hline Bem-Estar (O) & 2,81 & $3,20^{a}$ & 3,04 \\
\hline Conformidade $(\mathrm{O})$ & 1,88 & 2,09 & 2,11 \\
\hline Domínio (O) & $2,49 b c$ & 2,05 & 2,09 \\
\hline $\begin{array}{l}\text { Preocupação com a } \\
\text { Coletividade }(\mathrm{O})\end{array}$ & 2,19 & 2,14 & 2,21 \\
\hline Prestígio (O) & 1,69 & 1,90 & $2,09^{a}$ \\
\hline Realização (O) & 2,32 & 2,21 & 2,11 \\
\hline Tradição (O) & 3,36 & 3,31 & 3,42 \\
\hline
\end{tabular}

Fonte: Dados da pesquisa.

Pelos dados da Tabela 3, é possível constatar a existência de diferenças estatisticamente significativas entre as três gerações investigadas no que se refere às categorias de valores organizacionais "bem-estar", "domínio" e "prestígio".

Com relação ao valor "bem-estar", os dados revelam que a geração baby boomers o percebe em maior grau, comparativamente às gerações $\mathrm{Y}$ e X.

No que tange ao valor "domínio", constata-se maior sensibilidade da geração $X$ a essa prioridade axiológica, quando comparada às gerações Y e baby boomers.

Finalmente, quanto ao valor "prestígio", a geração baby boomers o percebe com mais intensidade que a geração $X$, e esta, por sua vez, o percebe mais que a geração $Y$. Quanto aos demais fatores, não foram observadas diferenças estatisticamente significativas.

A seguir, apresentar-se-ão as relações entre os construtos VP e VO, por par geracional investigado. 


\subsection{Valores pessoais e organizacionais por par geracional investigado}

Com base no conjunto dos dados coletados, foi possível verificar relações de conflito ou aderência entre cada um dos dez valores pessoais investigados e o escore médio dos valores organizacionais para cada grupamento geracional. Para tal análise, foi obtido um total de 80 comparações, considerando cada par geracional - X-BB, Y-BB e $X-Y$. Resultados da análise de cada uma dessas 80 relações indicam, para o par geracional $\mathrm{X}-\mathrm{BB}, 23$ diferenças entre os dez valores pessoais e o escore médio dos valores organizacionais do par, com destaque para diferenças significativas concentradas junto aos valores pessoais "autodeterminação", "benevolência", "hedonismo" e "tradição".

$\mathrm{Na}$ comparação envolvendo o par geracional Y-BB, foram registradas 33 diferenças, igualmente concentradas nos valores pessoais "autodeterminação", "benevolência", "hedonismo" e "tradição".

Quanto ao par geracional X-Y, por sua vez, os cálculos indicam diferenças estatisticamente significativas para 23 das 80 relações; desta feita, no entanto, com ampla concentração no valor pessoal "benevolência".

Quando consideradas as relações entre valores pessoais e organizacionais, a partir da relação entre os dez valores pessoais e cada um dos oito valores organizacionais investigados, foram analisadas 240 relações. Destas, somente 28 não revelaram diferenças estatisticamente significativas para algum par geracional.

Como resultado da análise de cada uma das 212 relações com diferenças estatisticamente significativas, foi possível identificar concentração das relações com os valores pessoais "autodeterminação", "benevolência", "hedonismo" e "tradição". Há que se enfatizar também que as diferenças estão localizadas em maior número (33) no par geracional Y-BB, podendo indicar maior dificuldade nas relações entre os representantes desse grupamento, se comparadas, por exemplo, ao par X-BB. 
Em que pese tal achado, os dados também apontam maior complexidade nas relações da geração $X$ com as gerações $Y$ e baby boomers. Tal dado muito provavelmente pode ser explicado pelo próprio caráter intermediário da geração X - posição de meio do "sandwich" -, com maiores demandas potenciais quanto a mediações tanto entre os novos valores em jogo, aportados pela geração $Y$, quanto entre aqueles transacionados pela geração ainda no poder, a baby boomers.

\section{Considerações finais}

A partir do conjunto dos dados obtidos, é possível concluir quanto à existência de diferenças estatisticamente significativas entre valores pessoais e organizacionais entre as diferentes gerações investigadas: baby boomers, $\mathrm{X}$ e $\mathrm{Y}$.

Dessa forma, em resposta à questão central deste estudo, podese indicar: 1) diferenças estatisticamente significativas entre as gerações baby boomers, $\mathrm{X}$ e $\mathrm{Y}$, no que se refere aos valores pessoais de poder, realização e universalismo; 2) diferenças estatisticamente significativas entre as gerações baby boomers, $\mathrm{X}$ e $\mathrm{Y}$ no que se refere aos valores organizacionais de bem-estar, domínio e prestígio; 3) diferenças estatisticamente significativas entre os pares geracionais $\mathrm{X}-\mathrm{BB}, \mathrm{Y}-\mathrm{BB}$ e $X-Y$, notadamente em relação aos valores pessoais de autodeterminação, benevolência, hedonismo e tradição, quando comparados ao conjunto dos valores organizacionais.

Assim sendo, cabe destacar maior ênfase por parte da geração $Y$ dos valores pessoais "hedonismo" e "realização", comparativamente a "benevolência", mais valorizada pelos representantes da geração baby boomers e X. Ademais, percebe-se que as gerações X e Y apresentamse mais orientadas a valores que privilegiam "abertura", "mudança" e "autopromoção", enquanto os representantes da geração baby boomers se orientam mais fortemente a valores como "autotranscendência" e "conservação". 
Nessa direção, cabe não perder de perspectiva o fato de os componentes da geração $Y$ encontrarem-se em pleno processo de inserção na vida profissional, com maiores expectativas e ímpeto quanto às suas possibilidades individuais, enquanto a geração baby boomers, pelo seu momento no ciclo de vida profissional, pode estar mais propensa a priorizar valores que extrapolam as dinâmicas intramuros das organizações. É possível, desse modo, que, ao se aproximarem da faixa etária da geração baby boomers, os membros da geração $Y$ venham a privilegiar valores como "autotranscendência" e "benevolência".

Em decorrência de tais achados, aponta-se para maior atenção às relações entre a geração X e as gerações $Y$ e baby boomers, notadamente ao se considerar a tendência de ampliação de representantes daquela primeira - com a aposentadoria da geração baby boomers - em posições organizacionais mais estratégicas, com maiores responsabilidades sobre a gestão dos elementos humanos e resultados organizacionais. Nesse sentido, políticas e práticas de gestão associadas ao desenvolvimento de competências, novos estilos de liderança, bem como iniciativas e programas mais sistêmicos de qualidade de vida, sentidos do trabalho e estresse ocupacional podem se configurar relevantes para a mitigação de impactos decorrentes das ambiguidades, conflitos e mal-estares associados.

Quantoà análise dos valores organizacionais, dados do conjunto dos grupos geracionais investigados apontam para menor ênfase em relação ao valor "tradição", assim como maior aderência dos três grupamentos geracionais pesquisados a valores associados à "preocupação com a coletividade". Concomitantemente, uma análise comparativa entre os grupamentos investigados aponta para maior ênfase das gerações $\mathrm{X} e$ Y a valores associados ao "bem-estar", "domínio" e "equilíbrio entre vida pessoal e profissional”, comparativamente à geração baby boomers.

Em suma, o conjunto dos dados permite constatar diferenças nas relações entre as distintas gerações investigadas que convivem ou poderão vir a conviver nos mesmos ambientes de trabalho. Porém, convém ressaltar que tais diferenças não se limitam à geração Y, como 
comumente enfatizado pela mídia e mesmo pela literatura especializada, mas ao conjunto dos grupamentos geracionais pesquisados, evidenciando a relevância de maior ênfase organizacional à gestão da diversidade, com o lidar com as diferenças.

Visam-se, a partir do aprofundamento das análises sobre os dados obtidos, novos elementos aos debates sobre o tema, propiciando um maior aprofundamento teórico dos estudos envolvendo a perspectiva geracional, manifesta na análise de seus valores pessoais e organizacionais, e o desenvolvimento de políticas e práticas de gestão de pessoas que considerem, com maior atenção, as diversidades inclusive geracionais - que caracterizam o contexto contemporâneo das organizações.

\section{Referências}

Arsenault, Paul M. (2004). Validating generational differences: a legitimade diversity and leadership issue. The Leardership \& Organization Development Journal, Ulster, NY, 25(2), 124-141.

Bagozzi, Richard P.; YI, Youjae; \& Philips, Lynn W. (1991). Assessing construct validity in organizational research. Administrative Science Quartely, Ithaca, NY, 36(3), 421-458.

Beck, Laura E. (2001). Development through the lifespan. Needham Heigts, MA: Allyn \& Bacon.

Cennamo, Lucy.; Gardner, Dianne. (2008). Generational differences in work values, outcomes and person - organization values fit. Journal of Managerial Psychology, Bingley, UK, 23(8), 891-906.

Chen, Po-Ju; Choi, Youngsoo. (2008). Generational differences in work values: a study of hospitality management. International Journal of Contemporary Hospitality Management, Bingley, UK, 20(6), 595615.

Chin, Wynne W. (1998). Issues and opinion on structure equation modeling. MIS Quarterly, Minneapolis, MN, 22 (2), vii-xvi. 
Coimbra, Rosangela Gamba C.; Schikmann, Rosane. (2001). A geração net. In: Encontro Da Associação Nacional de Programas de PósGraduação em Administração (ENANPAD), XV, Campinas. Anais ... Campinas: ENANPAD. CD-Rom.

Conger, Jay A. (2001). How 'Gen X' manage. 9-19. In: Osland, Joyce S.; Kolb, David; \& Rubin, Irwin M. (ed.). Organizational behavior reader. Upper Saddle River, NJ: Prentice-Hall, 9-19.

D'Amato, A..; Herzfeldt, R. (2008). Learning orientation, organizational commitment and talent retention across generations: a study of European managers. Journal of Managerial Psychology, Bingley, UK, 23(8), 929-953.

Dias, Devanir Vieira; Del Maestro Filho, A.; \& Moraes, Lúcio Flávio Renault de (2006). Valores organizacionais e modelos e práticas de gestão de pessoas nas empresas do setor siderúrgico do Estado de Minas Gerais. In: Encontro da Associação Nacional de Programas de Pós-Graduação em Administração (ENANPAD). Anais ... Salvador: ENANPAD. 1 CD-Rom.

Dries, Nicky; Pepermans, Roland; \& Kerpel, Evelien de (2008). Exploring four generations beliefs about career: is "satisfied" the new "successful"? Journal of Managerial Psychology, Bingley, UK, 23(8), 907-928.

Dunzer, Gustavo André; Melo, Eliana Medeiros Pereira; \& Melo, Joyce de Souza Cunha. (2008). Desafios da atração e retenção de talentos - estudo de caso da Petrobras. In: Encontro da Associação Nacional de Programas de Pós-Graduação em Administração (ENANPAD). Anais... Rio de Janeiro: ENANPAD. 1 CD-Rom.

Dutra, Joel Souza (2002). Gestão de pessoas. São Paulo: Atlas.

Dwyer, Rocky J. (2009). Prepare for the impact of the multi-generational workforce! Transforming Government: People, Process and Policy, Bradford, UK, 3(2), 101-110. 
Eyerman, Ron; Turner, Bryan S. (1998). Outline of a theory of generations. European Journal of Social Theory, London, UK, 1(4), 91-106.

Feather, Norman T. (1996). Values, deservingness, and attitudes toward high achievers: research on tall poppies. In: Seligman, Clive; Olson, James M.; \& Zanna, Mark P. (Ed.). The Ontario symposium: the psychology of values. Mahwah, NJ: Lawrence Eribaum Associates, $8(2), 215-251$.

Ferreira, Ássima Maria. (2008). Políticas e práticas de gestão de pessoas como fatores de retenção de jovens talentos: um estudo com trainees de empresa brasileira do setor siderúrgico. 2008. 220 f. Dissertação (Mestrado Profissional em Administração) - Programa de Pós-Graduação em Administração, Pontifícia Universidade Católica de Minas Gerais, Belo Horizonte.

Fornell, Claes; \& Larcker, David F. (1981). Evaluating structural equation models with unobservablevariables and measurement error. Journal of Marketing Research, Chicago, IL, 18(4), 39-50.

García-Lombardía, Pilar; Stein, Guido; \& Pin, José R. (2008). Generation Y: attracting and managing the millennials. Barcelona: IESE Business School.

Gouveia, Valdiney V. et al. (2001). A estrutura e o conteúdo universais dos valores humanos: análise fatorial confirmatória da tipologia de Schwartz. Estudos de Psicologia, Natal, RN, 6(2), 133-142.

Gursoy, Dogan; Maier, Thomas A.; \& Chi, Christina G. (2008). Generational differences: an examination of work values and generation gaps in the hospitality workforce. International Journal of Hospitality Management, Orlando, FL, 27(13), 448-458.

Halliman, Robert W. (1998). A coming of age: a comparison of organizational performance of baby boom CEOs to CEOs born prior to the baby boom era. Journal of Management History, Bingley, UK, 4(1), 68-74. 
Hankin, Harriet. (2004). The new workforce. New York: Amacom.

Hess, Narelle; Jepsen, Denise M. (2009). Career stage and generational differences in psychological contracts. Career Development International, Bingley, UK, 14(3), 261-283.

Kick, Fran. (2002). What makes kids kick: inspiring the millennial generation to kick-it-in! Centerville, $\mathrm{OH}$ : Instruction \& Design Concepts.

Kluckhohn, Clyde Kay Maben. (1951). Values and value orientations in the theory of action. In: Shils, Edward; Parsons, Talcott; \& Smelser, Neil J. (Ed.). Toward a general theory of action. Cambridge, MA: Harvard University Press, 388-433.

Kupperschmidt, Betty R. (2000). Multigenerational employees: strategies for effective management. The Health Care Manager, Philadelphia, PA, 19(1), 65-76.

Macky, Keith; Gardner, Dianne; \& Foryth, Stewart. (2008). Generational differences at work: introduction and overview. Journal of Managerial Psychology, Bingley, UK, 23(8), 857-861.

Maia, Lúcia de Fátima Rocha Bezerra; Oliveira, Joana D’Arc de; \& Ximenes, Verônica Morais. (2007). Impacto dos valores pessoais sobre o comportamento de cidadania organizacional dos funcionários do serviço de farmácia do Hospital Universitário Walter Cantídio da Universidade Federal do Ceará. In: Encontro da Associação Nacional de Programas de Pós-Graduação em Administração (ENANPAD). Anais... Rio de Janeiro: ENANPAD. 1 CD-Rom.

Martin, Carolyn A. (2005). What managers need to know about generation Y. Industrial \& Commercial Training, Bingley, UK, 37(1), 39-44.

Matsushita, Margarete. (2004). Gestão socialmente responsável: a influência dos valores dos gestores no papel de recursos humanos. 216 f. Dissertação (Mestrado em Administração de Empresas) Universidade Presbiteriana Mackenzie, São Paulo. 
Mattar, Fauze Najib. (2001). Pesquisa em marketing. 3. ed. São Paulo: Atlas.

McDonald, Kimberly S.; \& Hite, Linda M. (2008). The next generation of career success: implications for HRD. Advances in Developing Human Resources, Thousand Oaks, CA, 10(1), 86-103.

Meglino, Bruce M.; \& Ravlin, Elizabeth. C. (1998). Individual values in organizations: concepts, controversies, and research. Journal of Management, London, UK, 1(3), 351-389.

Mello, Newton Oller. (2004). A influência dos valores dos gestores na orientação para relações de confiança. 2004. 275 f. Dissertação (Mestrado em Administração) - Universidade Presbiteriana Mackenzie, São Paulo.

Mendonça, Helenides; \& Tamayo, Álvaro. (2005). Valores e retaliação organizacional. In: Tamayo, A.; \& Porto, J. B. (Orgs.). Valores e comportamento nas organizações. Petrópolis, RJ: Vozes.

Miguel, LílianAparecida Pasquini; \& Teixeira, Maria Luisa Mendes. (2009). Valores organizacionais e criação do conhecimento organizacional inovador. RAC - Revista de Administração Contemporânea, Curitiba, RS, 13(1), 36-56.

Montana, Patrick J.; \& Petit, Francis. (2008). Motivating generation X and $Y$ on the job and preparing $Z$. Global Journal of Business Research, Hilo, HL, 2(2), 139-148.

Netemeyer, Richard G.; Bearden, William O.; \& Sharma, Subhash. (2003). Scaling procedures: issues and applications. London: Sage Publications.

Oliveira, Áurea de Fátima; \& Tamayo, Alvaro. (2004). Inventário de perfis de valores organizacionais. RAUSP - Revista de Administração da Universidade de São Paulo, São Paulo, 39(2), 129-140. 
Pasquali, Luiz; \& Alves, Amélia Regina. (2004). Validação do portraits questionnaire: PQ de Schwartz para o Brasil. Avaliação Psicológica, Porto Alegre, RS, 3(2), 73-82.

Porto, Juliana Barreiros. (2005). Mensuração de valores no Brasil. In: Tamayo, Álvaro; Porto, Juliana Barreiros (Org.). Valores e comportamento nas organizações. Petrópolis, RJ: Vozes.

Porto, Juliana Barreiros; \& Tamayo, Álvaro. (2002). Prioridades axiológicas e regiões brasileiras: preditores de civismo nas organizações. Psicologia Organizações e Trabalho, Brasília, DF, 2(1), 31-61.

Richardson, Roberto Jarry. (2008). Pesquisa social: métodos e técnicas. São Paulo: Atlas.

Rohan, Meg J. (2000). A rose by any name? The values construct. Personality and Social Psycholoy Review, Mahwah, NJ, 4(3), 255-277.

Rokeach, Milton. (1973). The nature of human values. New York: Free Press.

Santos, Neusa Maria Bastos Fernandes dos; Abrahim, Gisele Seabra. (2008). A influência dos valores pessoais na determinação das âncoras de carreira. In: Encontro da Associação Nacional de Programas de Pós-Graduação em Administração (ENANPAD). Anais... Rio de Janeiro: ENANPAD. 1 CD-Rom.

Sarsur, Amyra Moyzez; Pedrosa, Rosangela Rezende; \& Sant'Anna, Anderson de Souza. (2003). Onde estão os talentos? Onde está a gestão de recursos humanos? In: Encontro da Associação Nacional de Programas de Pós-Graduação em Administração (ENANPAD). Anais... Atibaia, SP: ANPAD, 1 CD-Rom.

Schwartz, Shalom. (1999). A theory of cultural values and some implications for work. Applied Psychology: An International Review, Oxford, MA, 48(1), 23-47. 
Schwartz, Shalom. (1992). Universals in the content and structure of values: theoretical advances and empirical tests in 20 countries. In: ZANNA, MarKk(Org.). Advances in experimental social psychology. Orlando, FL: Academic, 25(1), 1-65.

Schwartz, Shalom. (2005a). Valores humanos básicos: seu contexto e estrutura intercultural. In: Tamayo, Álvaro; \& Porto, Juliana Barreiros (Org.). Valores e comportamento nas organizações. Petrópolis, RJ: Vozes, 21-55.

Smith, J. Walker; \& Clurman, Ann S. (1997). Rocking the ages. New York: Harper Business Press.

Strauss, William; \& Howe, Neil. (1997). The fourth turning. New York: Broadway Books.

Sullivan, Sherry E. et al. (2009). Using the kaleidoscope career model to examine generational differences in work attitudes. Career Development International, Bingley, UK, 14(3), 284-302.

Tamayo, Álvaro et al. (1998). Prioridades axiológicas, tempo de serviço e cidadania organizacional. Psicologia: Teoria e Pesquisa, Brasília, DF, 14(1), 45-50.

Tamayo, Álvaro. (2002). Exaustão emocional no trabalho. Revista de Administração, São Paulo, v. 37(2), p. 26-37.

Tamayo, Álvaro. (2005). Impacto dos valores pessoais e organizacionais sobre o comprometimento organizacional. In: Tamayo, Álvaro; \& Porto, Juliana Barreiros (Org.). Valores e comportamento nas organizações. Petrópolis, RJ: Vozes, p. 160-186.

Tamayo, Álvaro. (2000). Prioridades axiológicas e satisfação no trabalho. Revista de Administração, São Paulo, 35(2), 37-47.

Tamayo, Álvaro. (2001). Prioridades axiológicas, atividade física e estresse ocupacional. Revista de Administração Contemporânea, Curitiba, RS, 5(1), 127-147. 
Tamayo, Álvaro; Mendes, Ana Magnólia; Paz, Maria das Graças Torres da. (2000). Inventário de valores organizacionais. Estudos de Psicologia, Brasília, DF, 5(2), 289-315.

Toledo, Samanta. (2006). Carreira e Identidade: reflexos das exigências mercadológicas na vida pessoal e profissional dos jovens executivos de empresas multinacionais. In: Encontro da Associação Nacional de Programas de Pós-Graduação em Administração (ENANPAD). Anais... Salvador: ENANPAD. 1 CD-Rom.

Veloso, Elza Fátima Rosa; Dutra, Joel Souza; \& Nakata, Lina Eiko. (2008). Percepção sobre carreiras inteligentes: diferenças entre as gerações Y, X e baby Boomers. In: Encontro Da Associação Nacional de Programas de Pós-Graduação em Administração (ENANPAD. Anais ... Rio de Janeiro: ENANPAD, 1 CD-Rom.

Westerman, James W.; \& Yamamura, Jeanne H. (2007). Generational preferences for work environment fit: effects on employee outcomes. Career Development Journal, Bingley, UK, 12(2), 150-161.

Wong, Melissa et al. (2008). Generational differences in personality and motivation. Journal of Managerial Psychology, Bingley, UK, 23(28), 878-890.

Wyatt, David. (1993). Out of the sixties: storytelling and the Vietnam generation. Cambridge, MA: University Press.

Yu, Hui-Chun; \& Miller, Peter. (2005). Leadership style: the X generation and baby Boomers compared in different cultural contexts. Leadership \& Organizations Development Journal, Bingley, UK, 25(1), 35-50.

Zemke, Ron; Raines, Claire; \& Filipczak, Bob. (2000). Generations at work: managing the clash of veterans, Boomers, Xers, and Nexter in your workplace. New York: Amacon. 